\title{
Article \\ Discrete Wavelet Transforms-Based Analysis of Accelerometer Signals for Continuous Human Cardiac Monitoring
}

\author{
Hany Ferdinando ${ }^{1,2, *(\mathbb{0}}$, Eveliina Seppälä ${ }^{1}$ and Teemu Myllylä ${ }^{1,3}$ \\ 1 Research Unit of Medical Imaging, Physics, and Technology, Oulu University, 90100 Oulu, Finland; \\ eveliina.seppala@oulu.fi (E.S.); teemu.myllyla@oulu.fi (T.M.) \\ 2 Department of Electrical Engineering, Petra Christian University, Surabaya 60236, Indonesia \\ 3 Optoelectronics and Measurement Technique Research Unit, Oulu University, 90570 Oulu, Finland \\ * Correspondence: hany.ferdinando@oulu.fi
}

check for updates

Citation: Ferdinando, H.; Seppälä, E.; Myllylä, T. Discrete Wavelet

Transforms-Based Analysis of Accelerometer Signals for Continuous Human Cardiac Monitoring. Appl. Sci. 2021, 11, 12072. https://doi.org/ 10.3390/app112412072

Academic Editor: Maurizio Varanini

Received: 1 November 2021

Accepted: 12 December 2021

Published: 17 December 2021

Publisher's Note: MDPI stays neutral with regard to jurisdictional claims in published maps and institutional affiliations.

Copyright: (c) 2021 by the authors. Licensee MDPI, Basel, Switzerland. This article is an open access article distributed under the terms and conditions of the Creative Commons Attribution (CC BY) license (https:// creativecommons.org/licenses/by/ $4.0 /)$.

\begin{abstract}
Measuring cardiac activity from the chest using an accelerometer is commonly referred to as seismocardiography. Unfortunately, it cannot provide clinically valid data because it is easily corrupted by motion artefacts. This paper proposes two methods to improve peak detection from noisy seismocardiography data. They rely on discrete wavelet transform analysis using either biorthogonal 3.9 or reverse biorthogonal 3.9. The first method involves slicing chest vibrations for each cardiac activity, and then detecting the peak location, whereas the other method aims at detecting the peak directly from chest vibrations without segmentation. Performance evaluations were conducted on signals recorded from small children and adults based on missing and additional peaks. Both algorithms showed a low error rate ( $15.4 \%$ and $2.1 \%$ for children/infants and adults, respectively) for signals obtained in resting state. The average error for sitting and breathing tasks (adults only) was $14.4 \%$. In summary, the first algorithm proved more promising for further exploration.
\end{abstract}

Keywords: accelerometers; biomedical signal processing; discrete wavelet transforms; seismocardiography (SCG); peak detection

\section{Introduction}

As the heart has a central role in human health, assessing its condition is a prime concern for healthcare professionals. The easiest way to do this non-invasively is by using a stethoscope to listen to the heartbeat on the chest. Sounds convey useful information about the functioning of the heart, allowing cardiologists to identify abnormalities. Recordings of heart sounds are referred to as phonocardiograms (PCGs). Generally, each beat consists of two sounds, S1 and S2, and various algorithms have been developed to analyze them.

Another non-invasive method is measuring the electrical activity of the heart, resulting in an electrocardiogram (ECG). In this technique, electrodes are placed around the chest and other anatomically specified parts of the body. To obtain a complete view of ECG signals, medical standard ECG measurements use 12 leads. Other applications, such as wearable ECG straps, make do with fewer electrodes, commonly three. ECG signals allow the study of heart rate variability (HRV), which represents variation in inter-beat intervals. HRV analysis has a wide application range, not only in the medical field, but also in engineering, including affective computing.

Cardiac activities cause corresponding mechanical movements and vibrations in skin. Usually, such movements are easy to sense above arteries in the neck, wrist, and chest. Pioneered by Baevski, accelerometer-based chest vibration measurement is known as seismocardiography (SCG) [1]. Similar to PCG, a typical accelerometer signal includes S1 and S2 waves of each cardiac cycle. In this study, we use S1' and S2' to indicate waves in the SCG signal to avoid confusion with S1 and S2 in the PCG signal.

Accelerometers provide simple and affordable instrumentation, but the user must deal with several challenges; for example, if subjects move during measurements, the 
accelerometer will capture these motions. Since the accelerometer is very sensitive, even small environmental vibration may induce noise [2]. Other challenges include respiration and coughing.

Measuring adults in the clinical setting is not very difficult, because the patient can be instructed to stay still during the measurement. With infants and small children, however, this may prove more of a challenge. Another demanding task is measuring subjects during normal daily activities, such as walking and running, because motion artefact corrupts the signals.

Considering several important fiducial points, such as aortic valve opening (AO) and closure (AC), SCG holds great potential for clinical applications, including the assessment of myocardial contractility. It provides a non-invasive estimate of hemodynamic parameters [3] and left ventricular performance in patients with cardiac disease via systolic time intervals [1] as well as a non-invasive monitoring of stroke volume and cardiac time intervals [4]. Moreover, SCG can be used to estimate pre-ejection period (PEP) [5]. Examples such as these provide evidence that the method offers great promise. In addition, it complements ECG by providing information about the mechanical behavior of the heart [6].

Figure 1 shows a typical SCG signal measured from the chest; corresponding signals can also be harvested with an accelerometer above the carotid artery. Chuo et al. labeled $\mathrm{S}^{\prime}$ and S2' as the systolic and diastolic phase [7], respectively, while AO and AC represent the peak of the systolic and diastolic phase.

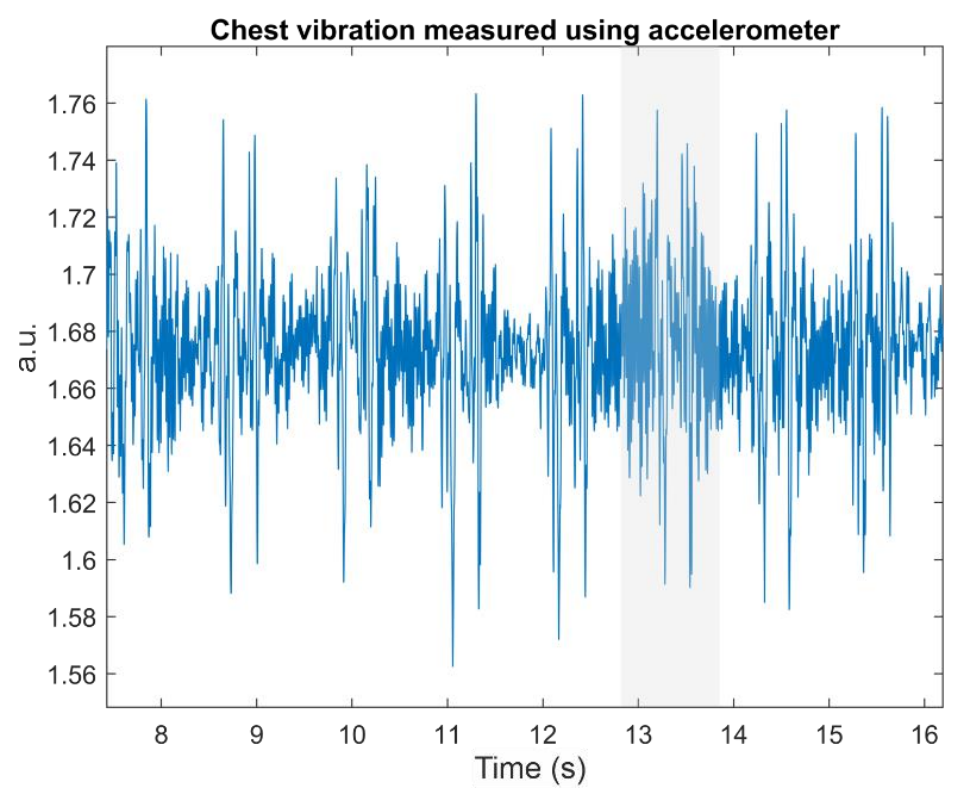

Figure 1. Typical SCG signal measured from the chest. Each heartbeat consists of $\mathrm{S}^{\prime}{ }^{\prime}$ and $\mathrm{S} 2^{\prime}$, which are close to each other, like those in PCG. Although the signal under the shaded area, around 13-14 s, is corrupted, both $\mathrm{S}^{\prime}$ and $\mathrm{S} 2^{\prime}$ are still visible.

In Figure 1, we can clearly see that, although the $\mathrm{S1}^{\prime}$ and $\mathrm{S2}^{\prime}$ waves around the 13th second are corrupted (see the shaded area in Figure 1), both waves are still visible. Analogous to S1 and S2, S1' tends to exhibit a larger amplitude than S2'. Figure 1 also shows that a series of $\mathrm{S}^{\prime}$ followed by $\mathrm{S} 2^{\prime}$ builds the whole cardiac activity signal. The distance between $\mathrm{S1}^{\prime}$ and $\mathrm{S}^{\prime}{ }^{\prime}$ within the same cardiac cycle is shorter than that of $\mathrm{S} 2^{\prime}$ from the current beat and $\mathrm{S}^{\prime}$ from the next beat. We can use these facts to get individual pairs of $\mathrm{S}^{\prime}$ and $\mathrm{S2}^{\prime}$. This can be easily done with a low heart rate.

A series of AO timings can be used to calculate heart rate. Furthermore, researchers also use AO to estimate blood pressure based on pulse transit time (PTT) between biosignals, such as ECG, and accelerometer data from, for example, the carotid artery on the neck [8]. 
Thus, $\mathrm{AO}$ detection is of high interest and requires robust algorithms to handle noisy signals resulting from motions and vibrations caused by factors other than cardiac activity.

A list of weaknesses of SCG, compiled by Zanetti and Tavakolian, includes complex waveforms that are difficult to interpret [1]. SCG signals also vary by age, sex, heart condition and body position [5,9]. However, these facts open various opportunities to develop new signal processing algorithms and improve sensor and instrumentation technology.

Researchers around the globe have been working to find good methods to detect AO automatically. The easiest way to detect AO is using ECG, especially R-peak, as a guideline. Khosrow-Khavar et al. evaluated four different methods, namely, cardiac sound characteristic waveform (CSCW), Shannon, absolute and Hilbert, to get the envelope of SCG signals by utilizing their high-frequency $(>20 \mathrm{~Hz})$ and low-frequency $(<30 \mathrm{~Hz})$ parts [10]. Mafi, in turn, used modified signal energy thresholding, empirical mode decomposition (EMD) and empirical wavelet transform to detect AO [11]. Shafiq et al. measured SCG from adults in a supine position without any tasks and aimed to detect peaks in the systolic phase [12].

The main problem with the abovementioned methods is that they rely on the availability of ECG signals. However, ECG sensor instrumentation is more demanding than that of SCG. It usually uses several electrodes placed on the subject's body with good skin-electrode contact. If the main goal is to measure HRV or heartrate (HR), then accelerometers provide easy instrumentation at an affordable price. This calls for methods to detect various important peaks in SCG in the absence of ECG.

Attempting to exclude ECG signals from AO detection, researchers have used cardiac cycle envelope (CCE) detection estimated from SCG as a guide [13-16] in combination with signals measured by a 3D gyroscope [17], a machine learning algorithm [18], a matchfilter [8] and simple moving average (SMA) [19]. Although the results are promising, the proposed algorithms suffer from various parameter settings and only work well for good SCG signals, characterized by low noise, clear separation between adjacent cardiac beats and higher peak in the systolic than in the diastolic phase. In addition, most of these algorithms were evaluated against SCG signals measured from mainly healthy adults in a supine position, thereby limiting their applicability. Some algorithms were also too complex and might not be suitable for real-time analysis.

In this paper, we proposed and evaluated two methods to detect $\mathrm{AO}$ from only SCG signals for real-time application. The methods relied on the discrete wavelet transform (DWT) either to guide SCG segmentation for each cardiac cycle, prior to AO detection, or to find AO detection directly without segmentation. SCG signals were measured from both children/infants and adults at resting state by using an accelerometer placed on the chest. In addition to a normal resting situation, the developed algorithms were tested using various breathing tasks (adults only).

\section{Materials and Methods}

\subsection{Data Acquisition}

In the data acquisition, two types of accelerometers were used. In the first data acquisition, measured by Seppälä [20], accelerometer model LIS344ALH (high performance 3 -axis $\pm 2 / \pm 6$ g ultra-compact linear accelerometer from STMicroelectronics) was placed on the chest of test subjects ( $n=7,32 \pm 25$ months) to measure chest vibrations, while they were sleeping (see Figure 2, sampled at $5 \mathrm{kHz}$ ). A consent letter was signed by the parents, giving permission to use their children's data. Strong, skin-friendly tape was used to secure the sensor in its position during the measurement. In addition, the sensor cable was secured with tape to minimize noise. As the measurements did not include ECG to exactly mark the peak of $\mathrm{S1}^{\prime}$, this was done by human visual inspection. 


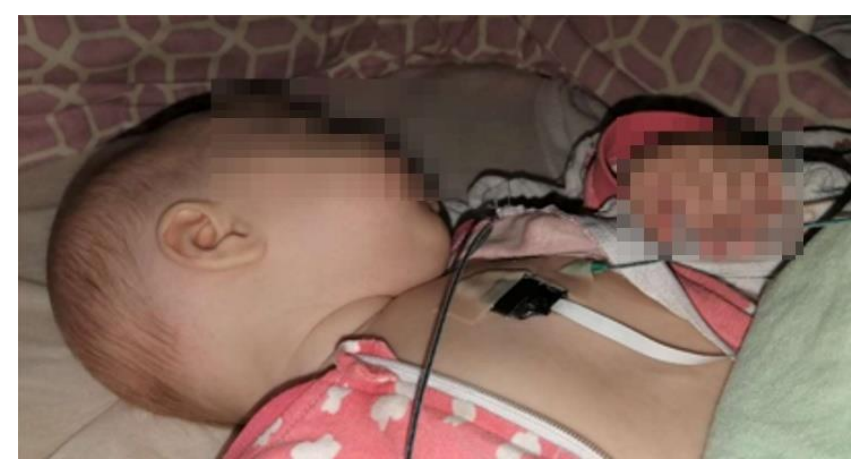

Figure 2. Measurement setup for small children and infants [20]. The accelerometer was placed on the chest of the subjects after they fell asleep. As some subjects moved in their sleep, they induced motion artefacts to the recorded signals.

In the second data acquisition, we used a single-axis opto-mechanical accelerometer, which is designed to be used in magnetoencephalography (MEG) and in magnetic resonance imaging (MRI), described in [21,22]. In addition, ECG was measured using 3 electrodes. Data was recorded from a group of university students $(n=9,28 \pm 5$ years old) and a consent letter was signed by each subject, giving permission to use their data. This group was asked to perform certain tasks during the measurements, i.e., Sitting, Breath Hold, Valsalva, and Deep Breathing. In addition to these tasks, the subjects were recorded at rest in a supine position. For both children/infants and adults, the accelerometer was placed on the lowest part of the sternum bone [11].

We were interested in evaluating the performance of the proposed algorithms during different breathing tasks, designed to induce motion artefacts due to chest movement.

- Sitting: subjects sat on a chair with no back support for two minutes. This position forces them to stabilize themselves and may induce motion artefacts.

- $\quad$ Breath Hold: subjects held their breath for $32 \mathrm{~s}$ and breathed normally for $88 \mathrm{~s}$. This series of breathing patterns was repeated three times.

- Valsalva: subjects blew into a breathing tube for $30 \mathrm{~s}$ followed by a $30 \mathrm{~s}$ rest. The procedure was repeated three times.

- Deep Breathing: subjects inhaled for five seconds and exhaled for five seconds. This task was repeated six times.

\subsection{Signal Enhancement}

We can easily segment PCG signals based on the cardiac cycle, because the distance between $\mathrm{S} 2$ of the $\mathrm{n}$-th beat and $\mathrm{S} 1$ of the $(\mathrm{n}+1)$-th beat is clear. SCG signals, however, may be another matter, when the amplitudes of $\mathrm{S}^{\prime}$ and $\mathrm{S} 2^{\prime}$ are close to each other and a high HR makes the $\mathrm{S}^{\prime}$ of the next beat move closer to the $\mathrm{S}^{\prime}{ }^{\prime}$ of the current one. For this reason, signal enhancement is essential.

We used an empirical mode decomposition (EMD)-based method for signal enhancement [23]. Unfortunately, this method was not suitable for real-time application, although it resulted in good, enhanced signals with clear separation for each cardiac cycle. For this reason, we left out this method and used a 5th-order Butterworth bandpass filter at $20-50 \mathrm{~Hz}$ to remove high frequency noise, possible baseline wandering, and other low frequency oscillations. Figure 3 compares raw and enhanced signals using the two methods. 

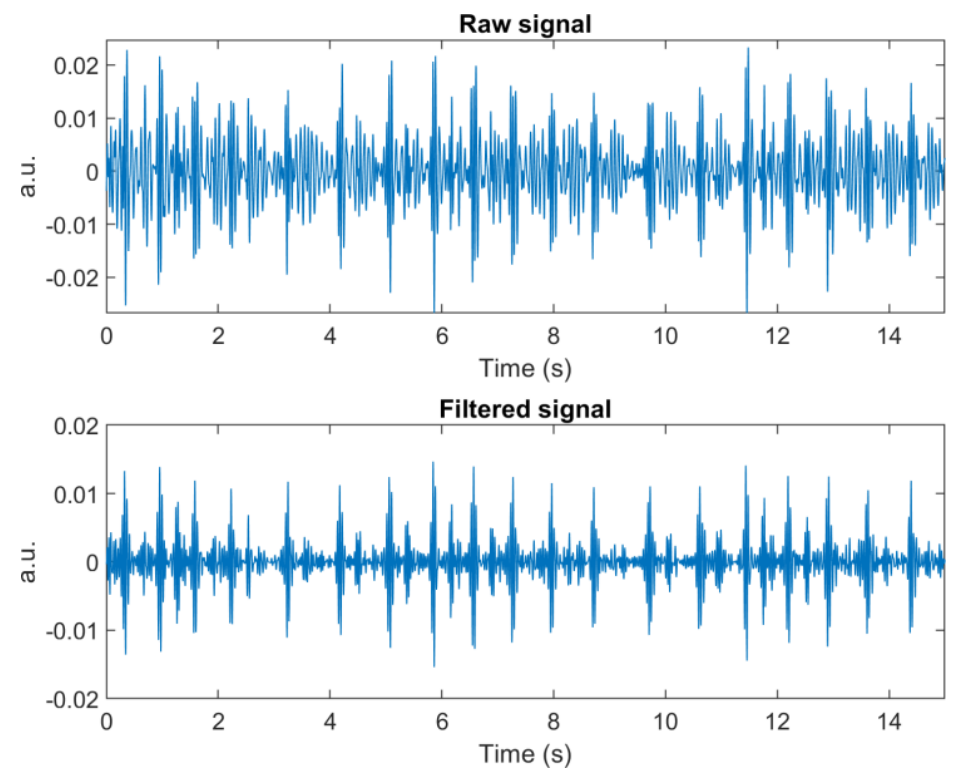

Figure 3. Raw signals (top) contained high frequency noise that blurred the systolic and diastolic parts in each cardiac cycle. Applying BPF with cut-off frequencies of 20 and $50 \mathrm{~Hz}$ improved the signal quality by removing high frequency noise and relatively low frequency oscillation between the two AOs (bottom).

\subsection{Method \#1: Slice and Detect}

Slice and Detect mimics the Zienkiewicz method [8] by segmenting signals based on the cardiac cycle, and then finding the peak of $S 1^{\prime}$ for each segment based on a specific window search area. This study proposes another method to replace the template filter method, based on utilising DWT for signal decomposition to guide the segmentation process. Algorithm 1 shows the pseudocode of Slice and Detect.

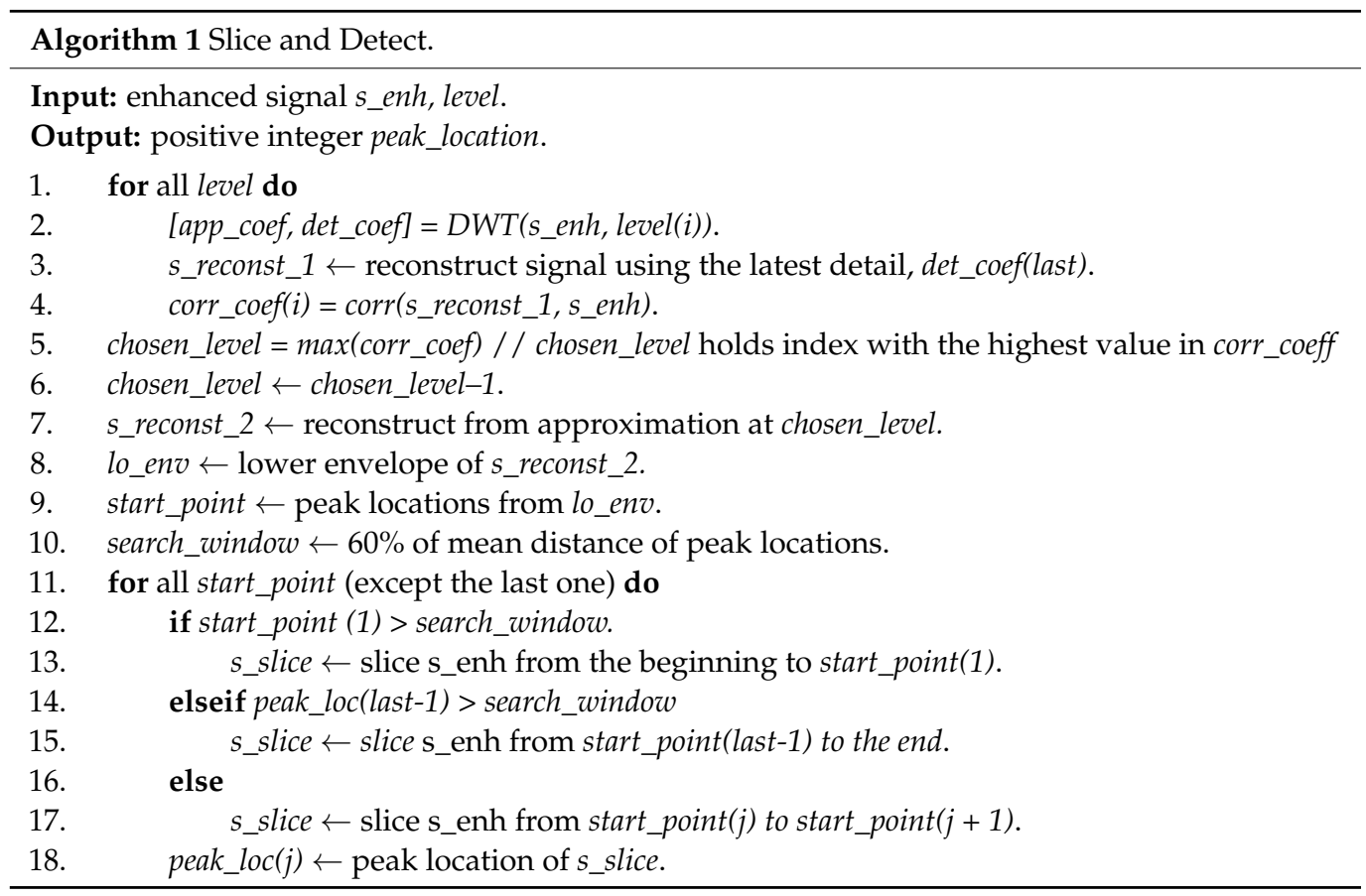

Appropriate mother wavelets are selected based on their similarity to the shape of $\mathrm{S1}^{\prime}$ and S2' $[24,25]$. Potential candidates include narrow symmetric wavelets, such as Symlets, 
Coiflets, Biorthogonal, Reverse biorthogonal, and Fejer-Korovkin wavelets in addition to wide asymmetric ones, such as Daubechies wavelets.

Another critical factor in wavelet decomposition is the number of the decomposition level. We do not want to lose all oscillations, because they are necessary for detecting the peak of $\mathrm{S1}^{\prime}$. For this reason, we calculate the correlation of the latest Detail, for example, D8 from the eight-decomposition level and the input signal (lines 1 to 4 in Algorithm 1). The level with the highest correlation minus one is selected for that segment (lines 5 to 6 in Algorithm 1).

Next, the lower envelope of the Approximation signal of the selected level is calculated, along with its peaks. These peaks indicate the locations for slicing the recorded signal into individual chest vibrations (lines 7 to 9 in Algorithm 1). Hence, they represent the start of the $\mathrm{S} 1^{\prime}$ wave.

The search window was defined as $60 \%$ of the mean of the distance between two adjacent starts of individual chest vibrations (line 10 in Algorithm 1). The main idea is to include the whole S1' wave. Hence, we applied an adjustable search window to accommodate heart rate variation. AO detection utilises an enhanced signal, instead of the original one.

Vibration on the edge needs special attention since the algorithm processes signals segment-by-segment to enable a real-time application. It is possible to have one chest vibration at the beginning of the signal, before the first detected starting point. To solve this process, the algorithm searches for the peak of a chest vibration, when the distance of the first starting point from the beginning of the segmented signal is greater than the search window (lines 12 to 13 in Algorithm 1).

Searching for the peak of the last chest vibration may generate an error if the search window is beyond the length of the current segment. To counter that, we calculate the mean of the distance between the starting point and its corresponding peak. When the last starting point is located further than the edge of the segment, the algorithm will search for a peak (lines 14 to 15 in Algorithm 1). Figure 4 presents Algorithm \#1, including signal enhancement, in a block diagram.

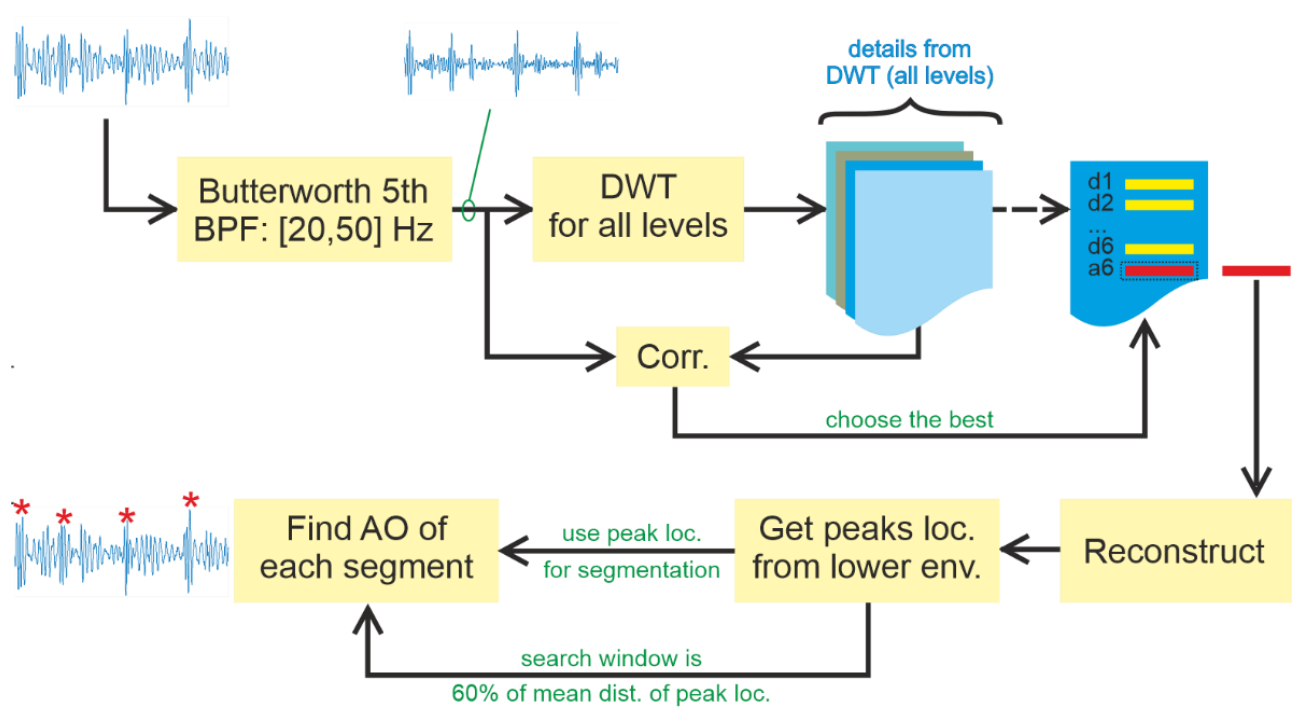

Figure 4. Both signal enhancement and Algorithm \#1 are presented as a block diagram. The asterisks in the figure represent the detected $\mathrm{AO}$ from the algorithm.

\subsection{Method \#2: Detect}

Detect finds peaks without signal segmentation. As a result, it must adopt a different approach to the task. This study proposes using maximum heart rate based on the age of the subject. To estimate maximum heart rate, researchers have introduced various models. A popular one is that of Steven Creeks [26] (see (1)). Among others, it has been utilised by 
Lauhkonen et al. in a study involving 166 children [27]. Wu et al. studied a bone marrow collection from 30 male subjects ( $40-65$ years, BMI less than $35 \mathrm{~kg} / \mathrm{m}^{2}$, type 2 diabetes) and 30 controls [28]. Sporis et al. evaluated various models on more than 500 military personnel and found that Creek's model provided a good correlation with the maximum heart rate, maxHR, obtained in a graded exercise test [29];

$$
\operatorname{maxHR}=205-\text { age } / 2,
$$

where age is expressed in years.

Maximum HR results in minimal RR intervals (minRRI), which can be used to correct errors. Wrongly detected peaks may arise from various sources, such as noise and oscillation characteristics around target peaks. Three measures were derived from minRRI: minimum peak distance for envelope finding ( $\operatorname{minDist} E n v)$, minimum peak distance for peak detection (minDistPeak) and span (correctionSpan) to the right and left for peak correction (lines 1 to 5 in Algorithm 2). They were empirically derived from experiments.

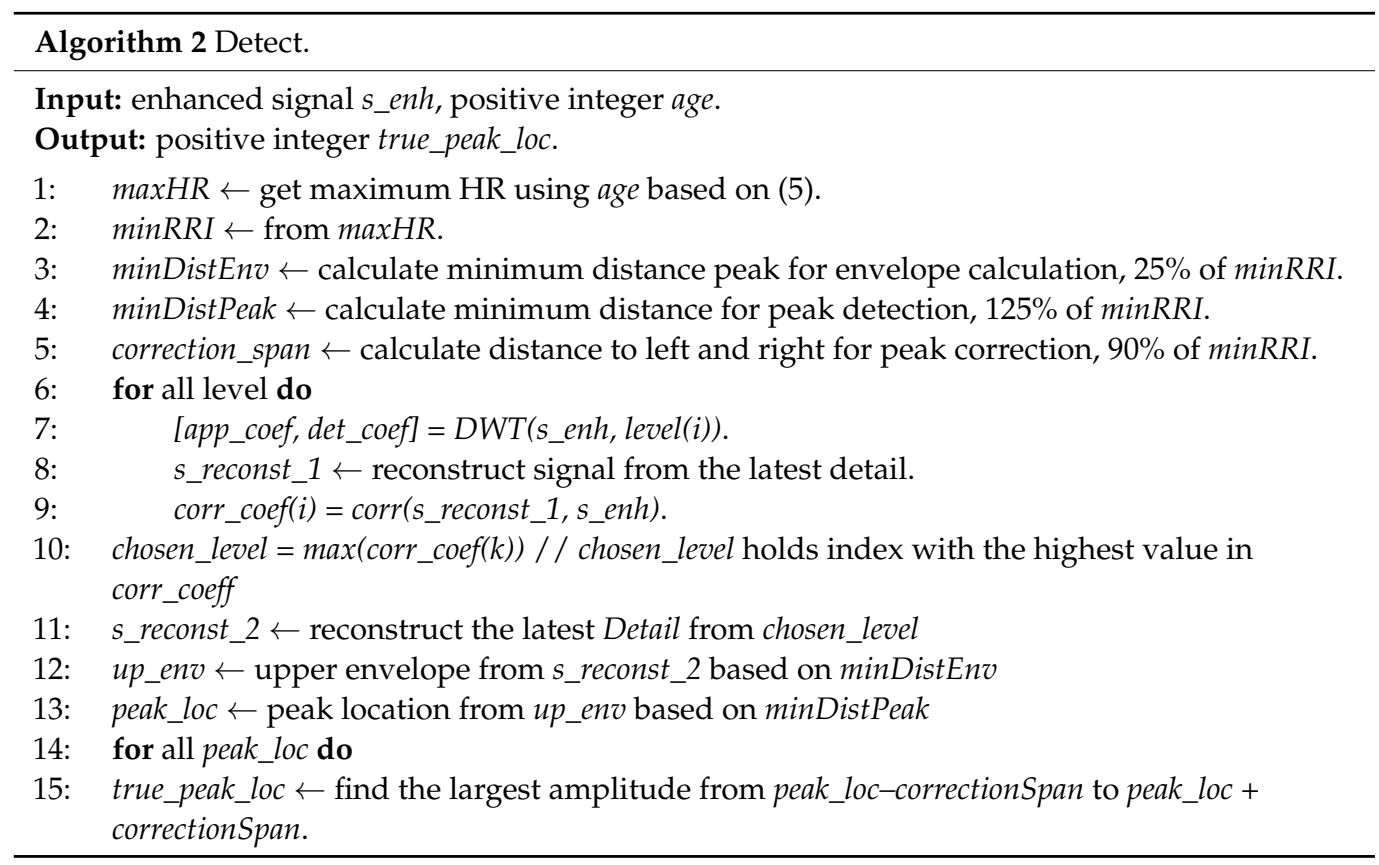

Detect starts with the application of DWT to the signal, be it whole or segmented, at a specific level, as in Slice and Detect (lines 6 to 11 in Algorithm 2). An upper envelope of the latest Detail from the selected level is generated based on its peaks, which are separated by at least minDistEnv (line 12 in Algorithm 2).

This envelope is then searched for its peaks. The distance between adjacent peaks must be at least minDistPeak (line 13 in Algorithm 2). These peaks point to systolic peak candidates of the original signal. Since getting the true systolic peaks cannot be guaranteed, they should be verified by finding the largest amplitude around the detected location. The width of the search area is limited by correctionSpan (lines 14 to 15 in Algorithm 2). Figure 5 presents Algorithm \#2, including signal enhancement, in a block diagram. 


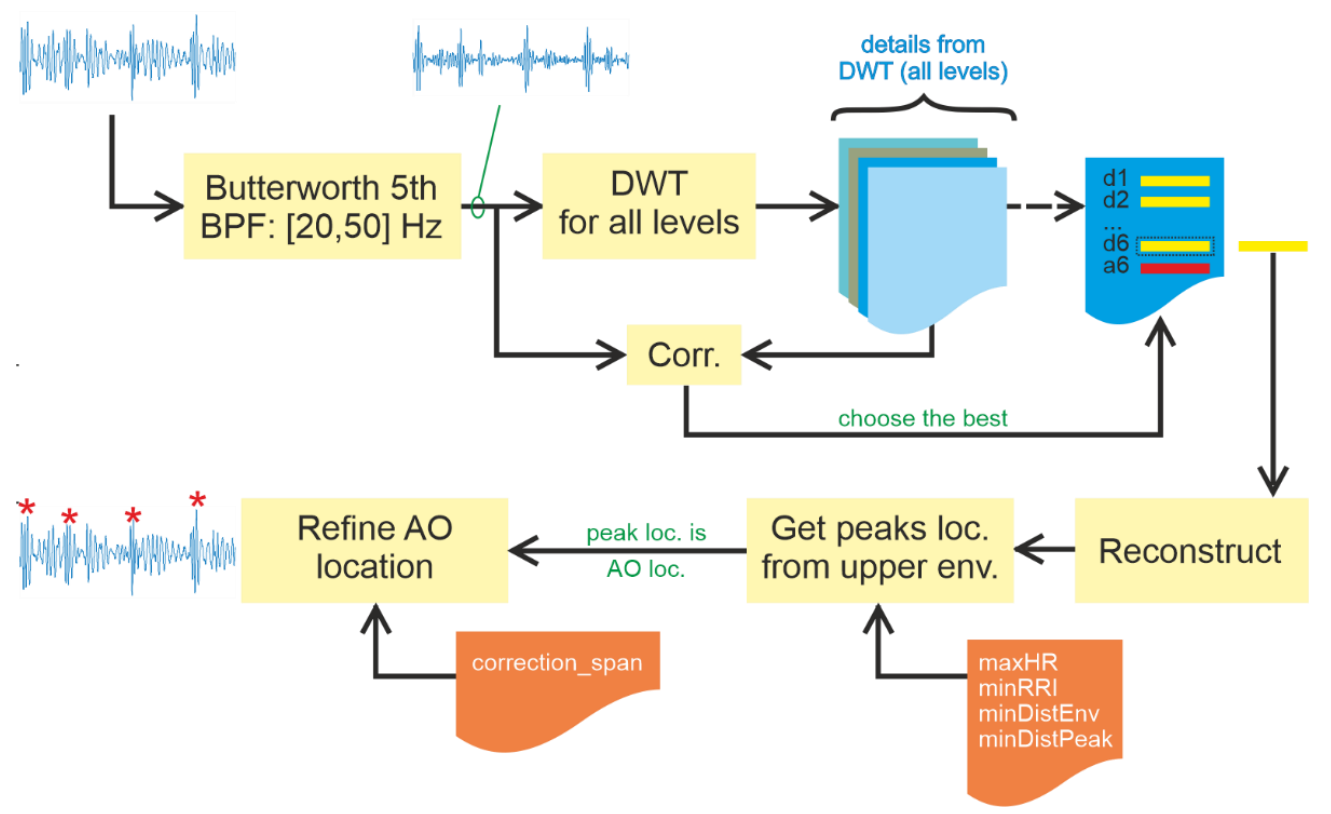

Figure 5. Both signal enhancement and Algorithm \#2 presented as a block diagram. The asterisks in the figure represent the detected $\mathrm{AO}$ from the algorithm.

\subsection{Analysis Method}

We used the R-peaks of the ECG signal as the ground truth to evaluate our proposed algorithms. However, signals from Group \#1 have no ECG signals. For this reason, manual annotation was used to provide ground truth for this group. In some cases, it was challenging to find the correct $\mathrm{AO}$ peaks, because infants and children have a higher heartrate than adults and systolic peaks were not always higher than diastolic ones. Segments containing these problems were discarded from analysis.

In the SCG from children/infants, we only measured accelerations perpendicular to the chest, which was sufficient for the analysis model. During peak detection, it is common to either miss or detect additional peaks. As these two errors are different, they will be handled separately. A missing peak is one that fails to be detected, whereas an additional peak is a fake peak found by the algorithm. To mark these errors, we evaluated the results manually. All computations were done using Matlab 2019b on a desktop computer with Intel(R) Core(TM) i7-8700 CPU @ 3.20 GHz, 16 GB RAM. For wavelet decomposition, we employed wavelet package decomposition 1D only.

\section{Results}

Experiments using all mother wavelet candidates revealed that only two mother wavelets gave satisfying results, namely, biorthogonal 3.9 and reverse-biorthogonal 3.9. It confirmed what Choudhary et al. found in their work [16]. Most other candidates failed to mark the boundary between two individual chest vibrations. As both biorthogonal 3.9 and reverse-biorthogonal 3.9 gave similar results, it is difficult to choose the best one. Figure 6 compares the results from symlet, biorthogonal, and reverse-biorthogonal. In addition, manual AO detection proved challenging, as signals from Group \#1 had no ECG signal. Khosrow-Khavar et al. have already addressed this challenge, even for adults [15]. They found that $\mathrm{S1}^{\prime}$ tends to have a higher amplitude than $\mathrm{S} 2^{\prime}$. However, in some children, both can have a similar amplitude, and sometimes the amplitude of S2' is larger than that of S1'. 

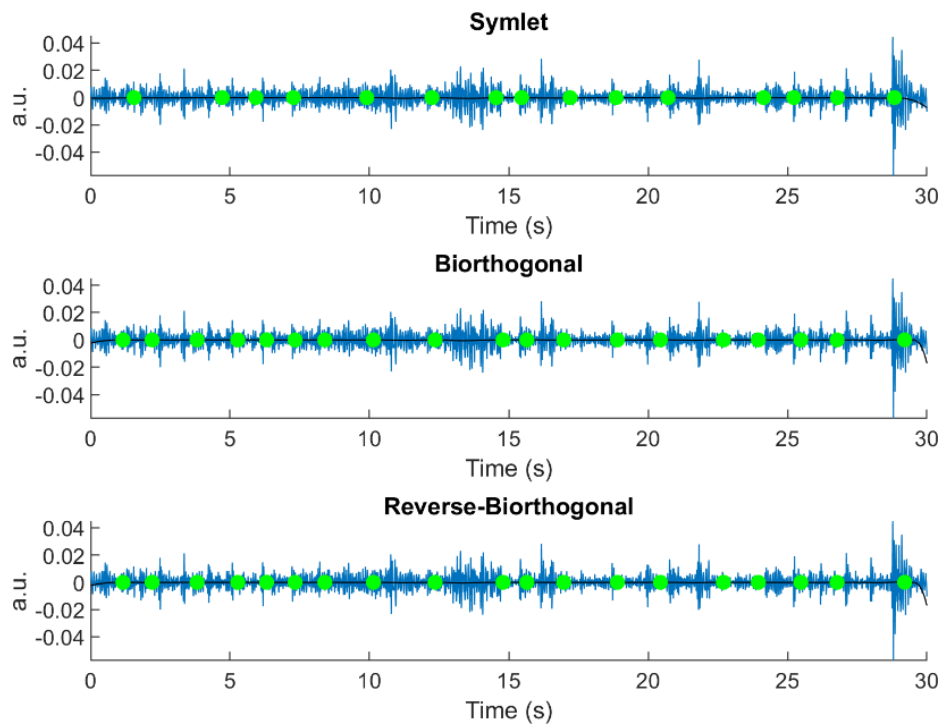

Figure 6. Marking the start of complex pulses from Algorithm \#1: Slice and Detect using symlet, biorthogonal, and reverse-biorthogonal mother wavelets. The green dots show the start of each complex pulse. They also serve as the starting point to search for the AO peak in each cardiac cycle. It shows clearly that symlet missed most of the starting points.

Figure 7 displays results from Slice and Detect. The top plot shows the original signal with its detected peaks $(*)$ and starting points of the cardiac cycle $(\mathrm{o})$, whereas the bottom plot displays the Approximation signal and its lower envelope.
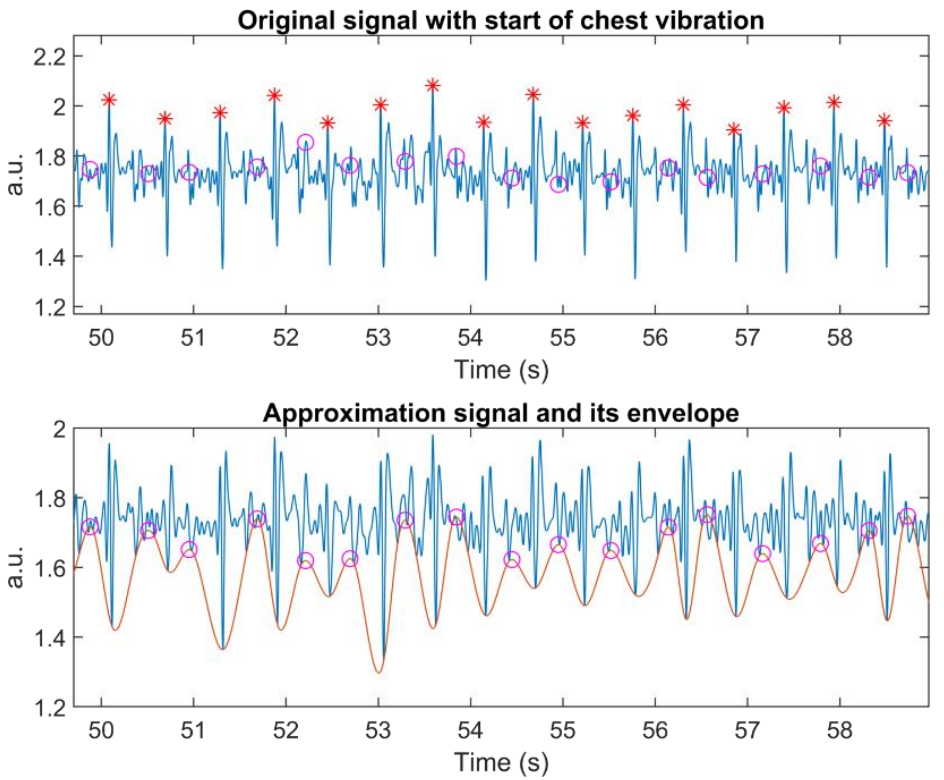

Figure 7. Result from Slice and Detect. Wavelet transform guides the determination of boundaries between individual chest vibrations by providing a peak from the envelope of the Approximation signal (o). The starting points were used to search for corresponding peaks in the enhanced signal, shown as asterisks $\left({ }^{*}\right)$ on top figure.

Figure 8 presents results from Detect, which detects peaks without slicing signals into individual segments based on the cardiac cycle.

Tables 1 and 2 show the error rate detection (\%) from Group \#1 and Group \#2, respectively, using both algorithms. Results shown in these tables came from the analysis using biorthogonal 3.9 only, as the performances from both biorthogonal and reversebiorthogonal showed no significant difference. Signals from three subjects were good 
in terms of low motion artefact level. However, the characteristics of SCG signals were atypical; $\mathrm{AC}$ was not always smaller than AO. As the measurements in Group \#1 did not include ECG, we have no reference for the real peaks. For this reason, they were marked as N/A (not available).
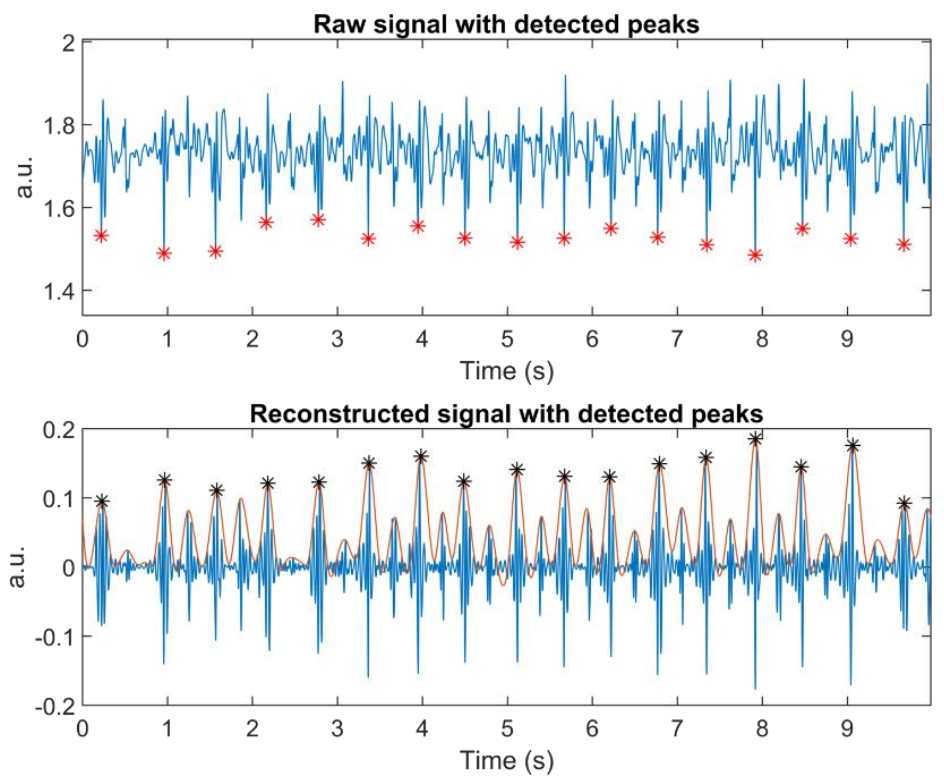

Figure 8. Result from Detect. Wavelet transform guides the determination of peak candidates $\left(^{*}\right)$ (bottom). The figure shows corrected peak locations $\left(^{*}\right)($ top). It is interesting to note here that Detect can follow variations in the inter-beat interval as shown in the beginning of the beat with a larger interval.

Table 1. Performance of Both Algorithms on Group \#1.

\begin{tabular}{ccccccc}
\hline \multirow{2}{*}{$\begin{array}{c}\text { Subject ID } \\
\text { (Months) }\end{array}$} & \multicolumn{3}{c}{ Slice and Detect } & \multicolumn{3}{c}{ Detect } \\
\cline { 2 - 6 } & Error $\mathbf{1}$ & Error 2 & Total & Error 1 & Error 2 & Total \\
\hline $1(5)$ & 3.5 & 1.4 & 4.9 & $\mathbf{2 . 1}$ & $\mathbf{1 . 1}$ & $\mathbf{3 . 2}$ \\
$2(8)$ & 32.6 & 3.4 & 36.0 & $\mathbf{9 . 5}$ & $\mathbf{0 . 3}$ & $\mathbf{9 . 8}$ \\
$3(26)$ & $\mathrm{N} / \mathrm{A}$ & $\mathrm{N} / \mathrm{A}$ & $\mathrm{N} / \mathrm{A}$ & $\mathrm{N} / \mathrm{A}$ & $\mathrm{N} / \mathrm{A}$ & $\mathrm{N} / \mathrm{A}$ \\
$4(26)$ & $\mathrm{N} / \mathrm{A}$ & $\mathrm{N} / \mathrm{A}$ & $\mathrm{N} / \mathrm{A}$ & $\mathrm{N} / \mathrm{A}$ & $\mathrm{N} / \mathrm{A}$ & $\mathrm{N} / \mathrm{A}$ \\
$5(32)$ & 16.2 & 9.4 & 25.6 & $\mathbf{1 4 . 3}$ & $\mathbf{0 . 4}$ & $\mathbf{1 4 . 7}$ \\
$6(53)$ & $\mathbf{1 7 . 6}$ & 15.3 & 32.9 & 25.8 & $\mathbf{8 . 1}$ & 33.9 \\
$7(76)$ & $\mathrm{N} / \mathrm{A}$ & $\mathrm{N} / \mathrm{A}$ & $\mathrm{N} / \mathrm{A}$ & $\mathrm{N} / \mathrm{A}$ & $\mathrm{N} / \mathrm{A}$ & $\mathrm{N} / \mathrm{A}$ \\
Avg. & 17.5 & 7.4 & 24.9 & $\mathbf{1 2 . 9}$ & $\mathbf{2 . 5}$ & $\mathbf{1 5 . 4}$ \\
\hline
\end{tabular}

$\overline{\mathrm{N}} / \mathrm{A}=$ not available, Avg. = average, Error 1 = error due to missing peaks, Error 2 = error due to additional peaks.

Table 2. Performance of Both Algorithms on Group \#2.

\begin{tabular}{ccccccc}
\hline \multirow{2}{*}{$\begin{array}{c}\text { Subject ID } \\
\text { (Months) }\end{array}$} & \multicolumn{3}{c}{ Slice and Detect } & \multicolumn{3}{c}{ Detect } \\
\cline { 2 - 7 } & Error $\mathbf{1}$ & Error 2 & Total & Error 1 & Error 2 & Total \\
\hline Rest & 5.4 & 1.7 & 7.1 & $\mathbf{1 . 9}$ & $\mathbf{0 . 2}$ & $\mathbf{2 . 1}$ \\
Sit & $\mathbf{1 4 . 5}$ & $\mathbf{0 . 2}$ & $\mathbf{1 4 . 7}$ & 17.8 & 2.6 & 20.4 \\
BH & $\mathbf{1 2 . 0}$ & $\mathbf{0 . 4}$ & $\mathbf{1 2 . 4}$ & 13.2 & 15.3 & 28.5 \\
Vals. & $\mathbf{1 4 . 9}$ & $\mathbf{2 . 5}$ & $\mathbf{1 7 . 4}$ & 12.9 & 12.9 & 25.8 \\
DB & $\mathbf{1 1 . 3}$ & $\mathbf{1 . 9}$ & $\mathbf{1 3 . 2}$ & 13.6 & 6.7 & 20.3 \\
Avg. & $\mathbf{1 1 . 6}$ & $\mathbf{1 . 3}$ & $\mathbf{1 2 . 9}$ & 11.9 & 7.5 & 19.2 \\
\hline
\end{tabular}

$\overline{\mathrm{BH}}=$ breath hold, Vals. = Valsalva, DB = deep breathing, Avg. = average, Err. 1 = error due to missing peaks, Err $2=$ error due to additional peaks. 


\section{Discussions}

Khosrow-Khavar et al. acknowledged that SCG signal suffers from its complexity [15]. Cardiac beat induces vibration to the skin and the accelerometer measures not only the pure skin vibration, but also its harmonics. The harmonic signals depend on the hardness of the tissue under the sensor. Hard tissue induces a lower damping factor than soft tissue.

In the absence of this damping factor, vibrations can be very noisy. However, if the damping factor is too high, the amplitudes of $\mathrm{S}^{\prime}$ and $\mathrm{S}^{\prime}$ are not clearly visible. As a countermeasure, a soft material can be inserted between the sensor and skin, providing a mechanical coupling [30]. Exploring these effects falls outside of the scope of this study, so future work will be needed to evaluate the proposed algorithms in this respect.

We also emphasize that accelerometer placement matters, as confirmed by Ashouri and Inan [5] and Chuo et al. [7]. We placed the accelerometer on the chest, typically above the sternum. However, in the case of Group \#1, the sensor was attached after the subjects fell asleep, and some sleeping body positions made placement in the planned position impossible. In Group \#2, the accelerometer could be placed on the target area, but, as the subjects were all adults, signal quality was affected by differences in chest area, caused by varying muscle thicknesses and fat deposition, for example.

Another problem was the accelerometer cable, especially in Group \#1. Children moved freely in their sleep and cable movements or bending sometimes affected signal quality.

Many researchers have presented useful algorithms for detecting peaks from the systolic and diastolic phases. The proposed algorithms rely on two assumptions based on PCG, (1) the peak of S1 is always larger than that of S2, and (2) there is an appropriate distance between $\mathrm{S} 2$ of the current cardiac cycle and $\mathrm{S} 1$ of the next one. However, the first assumption did not always hold for SCG, as shown by the results from Group \#1. The main drawback is these solutions often include ECG [10-12], which is not always available.

With the absence of an ECG signal to guide the determination of real peaks, performance evaluation in Group \#1 was quite challenging. With three out of seven subjects in Group \#1, the amplitude of S2' was not always smaller than that of $\mathrm{S1}^{\prime}$. Consequently, the peak position could not be determined.

Moreover, children and babies have a higher HR than adults, making the distance between $\mathrm{S}^{\prime}$ of the current cardiac cycle and $\mathrm{S1}^{\prime}$ of the next one even smaller. For this reason, no results were available for data analysis from three of the subjects in Group \#1.

The two assumptions discussed above were utilised in the algorithms presented here. Slice and Detect uses them to determine the start of each cardiac cycle, based on the peak of the lower envelope of Approximation signals. With an absolute AC larger than the absolute $\mathrm{AO}$, the shape of the envelope changed accordingly. Consequently, the algorithm missed the true starting point of the cardiac cycle. Further, this error propagated to the peak detection results.

The other algorithm used the assumptions to find prominent peaks in the envelope of signal reconstruction that leads to AO. Both algorithms work well when the absolute AC is less than the absolute $\mathrm{AO}$ within the same cardiac beat. Another important condition is that $\mathrm{S}^{\prime}$ and $\mathrm{S} 2^{\prime}$ must be sufficiently sharp. If these conditions are not fulfilled, the envelope and peak detection fail to produce optimal results.

In a resting state, both algorithms provided a low error rate for both groups. In this state, disturbance was almost non-existent, except for children and babies moving in their sleep. As a result, signal quality was good, and the low error rate makes sense.

For errors produced by the algorithms, additional peak error was always lower than missing peak error, except for Breath Hold and Valsalva for Detect in Group \#2. Both tasks force the subjects to compress their lungs, which may affect chest vibration characteristics. Interestingly, Slice and Detect showed a very low error rate in this situation. Perhaps DWT provided sufficient signal enhancement to provide a good separation between adjacent chest vibrations. Detect, on the other hand, enabled heartbeat detection from a very noisy signal (see Figure 9). 

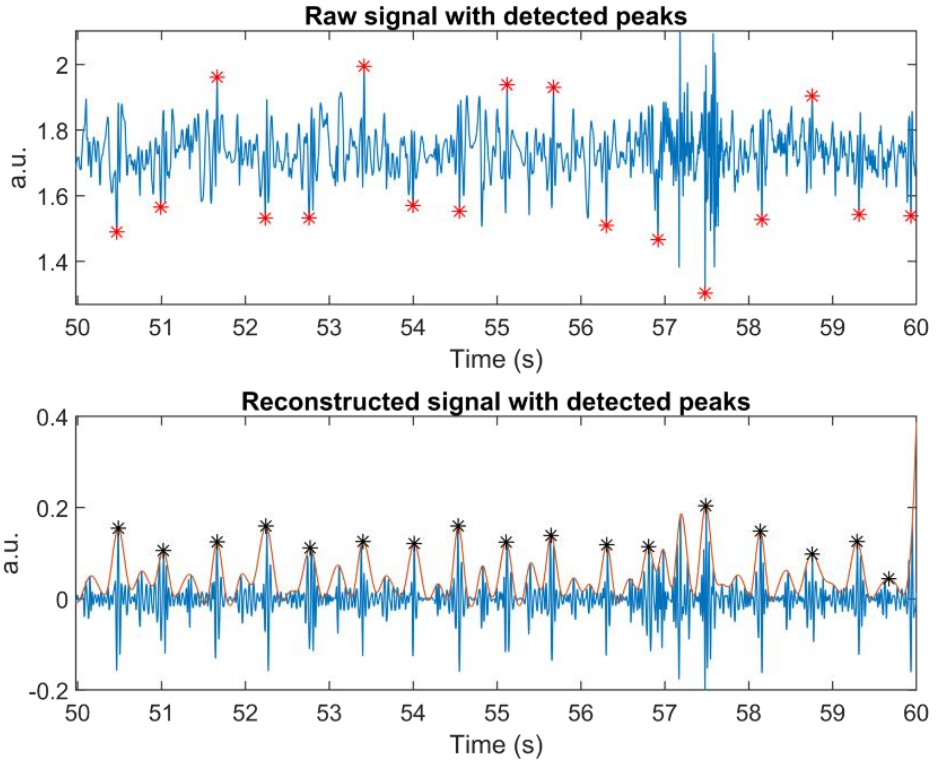

Figure 9. Detect uses wavelet transform to decompose signals based on the similarity of their shape with the mother wavelet. It offers the possibility to detect peaks, shown as asterisks that are hard to find even for a human.

In a resting state, Detect delivered fewer additional peaks in both groups. The most probable reason is that, in this state, signals were not disturbed by the various breathing tasks the subjects undertook. These tasks affect chest movement, inducing motion artefacts. As hypothesized above, DWT may provide a form of signal enhancement that offers a good separation between adjacent chest vibrations.

For Group \#1, Detect outperformed the other one in terms of average error rate detection. However, with only four subjects, it is difficult to draw meaningful conclusions from this table. That said, Detect showed promise, especially for monitoring children and babies.

During the Valsalva manoeuvre, both algorithms struggled to detect correct peak locations, when the heartbeat increased or dropped quickly. SCG signals were quite noisy during this task, mostly due to body movements. It would be impossible even for a human to pinpoint $\mathrm{AO}$ correctly without recourse to R-peaks.

Wavelet transform decomposes signals based on chosen mother wavelets and can be used to find parts of a signal that are similar in shape to the mother wavelet. Hence, it is a means of signal enhancement that can find buried information in a noisy signal. However, this ability failed to produce good results in Group \#2 during breathing tasks.

Using an envelope to find peak candidates in Detect came with a specific drawback: at the beginning of some segments, it failed to detect a prominent peak. If signal quality is good and the measurement is sufficiently long to produce signals with many prominent peaks, this problem can be neglected. However, for a short-time signal with poor quality, this is an important issue. Therefore, methods without envelope detection should be used or a robust parameter should be provided for peak detection, which is a subject for future work.

Choosing a decomposition level based on the correlation between the last detail reconstruction and the original signal proved successful. The nature of signal oscillation cannot be generalised, so each segment may require a different decomposition level. Our experiments showed that this level varies from five to eight.

Performance measurements in the adult dataset were easier, due to the availability of ECG, especially for signals with artefacts. The correct peak location always comes slightly after the R-peak of each heartbeat. Researchers define this time difference as pulse transit time (PTT), and it can be used, for example, for continuous estimation of blood pressure fluctuations [8] and the study of cerebral autoregulation [31]. 
Our data sets used two different accelerometers, ordinary MEMS for children/infants and opto-mechanical accelerometers for adults. Comparing their performances is only possible from the resting state of these two groups. However, nothing can be concluded thus far, because signals from adults are more controllable than those from children/infants. Consequently, another study must be conducted to make a more meaningful conclusion.

\section{Conclusions}

Two algorithms were proposed and evaluated to detect the peak of chest vibration measured by an accelerometer without ECG signals for real-time application. Both algorithms are based on DWT using either biorthogonal 3.9 or reverse biorthogonal 3.9 mother wavelets. Their performance was evaluated by peak detection error rate, defined as the ratio of missing and additional peaks to the ground truth. In addition to performance in resting state, they were evaluated against subject age and various breathing tasks. Both algorithms showed good performance for signals recorded in the resting state. Especially for the MRI compatible accelerometer, the algorithms are of high interest since during typical MRI measurement patients are in a supine position and usually resting. However, the breathing tasks challenged both, lowering their performances. Of the two, Slice and Detect looks more promising for further exploration. Because the accelerometers used in this study are meant for different patient monitoring applications and were used in different protocols, a meaningful comparison between their performance is not reasonable.

Determining decomposition level based on the correlation coefficient between the reconstructed and the original signal seems to provide a good solution, as opposed to using the same level for all. It gives the algorithms better ability to handle variations in the signal.

Both algorithms worked well in the resting state but struggled in the various breathing tasks. However, Slice and Detect looked promising for further investigation as DWT may provide a good separation between adjacent chest vibrations. Nevertheless, they ran into difficulties in the Valsalva manoeuvre, when the heartbeat raised and dropped quickly. Increasing the efficiency of the algorithms to handle various breathing tasks will be part of future work. This algorithm can be used to assess HR from SCG. Another application includes calculating pulse transit time based on another biosignal to study various cardiovascular parameters and cerebral autoregulation and circulation [23].

Future works include comparing the performance of both algorithms on different types of accelerometers and investigating dual tree complex wavelet for wavelet decomposition. To do it properly, signals must be measured simultaneously using the selected accelerometers. Other interesting investigations are studying the damping factor of soft materials placed under the accelerometer and employing dual tree complex wavelet analysis.

Author Contributions: Conceptualization all authors; data curation H.F. and E.S.; methodology H.F.; software H.F.; validation H.F. and E.S.; writing—original draft preparation H.F.; writing-review and editing all authors; visualization, H.F. and E.S.; funding acquisition and organizing the data collection T.M. All authors have read and agreed to the published version of the manuscript.

Funding: This research was funded by Academy of Finland, grant number 318347 and 335723.

Institutional Review Board Statement: The study was conducted according to the guidelines of the Declaration of Helsinki and approved by the Ethics Committee of Oulu University Hospital (approval number 296/2018 for children/infants and 53/2012 for adults).

Informed Consent Statement: Informed consent was obtained from all subjects and parents of the infants/children involved in the study.

Conflicts of Interest: The authors declare no conflict of interest.

\section{References}

1. Zanetti, J.M.; Tavakolian, K. Seismocardiography: Past, Present and Future. In Proceedings of the 2013 35th Annual International Conference of the IEEE Engineering in Medicine and Biology Society (EMBS), Osaka, Japan, 3-7 July 2013 ; pp. $7004-7007$.

2. Rai, D.; Thakkar, H.K.; Rajput, S.S.; Santamaria, J.; Bhatt, C.; Roca, F. A Comprehensive Review on Seismocardiogram: Current Advancements on Acquisition, Annotation, and Applications. Mathematics 2021, 9, 2243. [CrossRef] 
3. Tavakolian, K.; Blaber, A.P.; Ngai, B.; Kaminska, B. Estimation of Hemodynamic Parameters from Seismocardiogram. In Proceedings of the 2010 Computing in Cardiology, Belfast, UK, 26-29 September 2010; pp. 1055-1058.

4. Thakkar, H.K.; Sahoo, P.K. Towards Automatic and Fast Annotation of Seismocardiogram Signals Using Machine Learning. IEEE Sens. J. 2020, 20, 2578-2589. [CrossRef]

5. Ashouri, H.; Inan, O.T. Automatic Detection of Seismocardiogram Sensor Misplacement for Robust Pre-Ejection Period Estimation in Unsupervised Settings. IEEE Sens. J. 2017, 17, 3805-3813. [CrossRef]

6. Andreozzi, E.; Fratini, A.; Esposito, D.; Naik, G.; Polley, C.; Gargiulo, G.D.; Bifulco, P. Forcecardiography: A Novel Technique to Measure Heart Mechanical Vibrations onto the Chest Wall. Sensors 2020, 20, 3885. [CrossRef]

7. Chuo, Y.; Tavakolian, K.; Kaminska, B. Evaluation of a Novel Integrated Sensor System for Synchronous Measurement of Cardiac Vibrations and Cardiac Potentials. J. Med. Syst. 2011, 35, 445-455. [CrossRef]

8. Zienkiewicz, A. Blood Pressure Estimation Using Pulse Transit Time Models. Master's Thesis, University of Oulu, Oulu, Finland, 11 December 2017.

9. García-González, M.A.; Argelagós-Palau, A.; Fernández-Chimeno, M.; Ramos-Castro, J. A Comparison of Heartbeat Detectors for the Seismocardiogram. In Proceedings of the Computing in Cardiology 2013, Zaragoza, Spain, 22-25 September 2013; pp. 461-464.

10. Khosrow-khavar, F.; Tavakolian, K.; Blaber, A.P.; Zanetti, J.M.; Fazel-Rezai, R.; Menon, C. Automatic Annotation of Seismocardiogram With High-Frequency Precordial Accelerations. IEEE J. Biomed. Health Inform. 2015, 19, 1428-1434. [CrossRef] [PubMed]

11. Mafi, M. Signal Processing Methods for Heart Rate Detection Using the Seismocardiogram. Master's Thesis, University of Saskatchewan, Saskatoon, SK, Canada, 2015.

12. Shafiq, G.; Tatinati, S.; Veluvolu, K.C. Automatic Annotation of Peaks in Seismocardiogram for Systolic Time Intervals. In Proceedings of the 2016 38th Annual International Conference of the IEEE Engineering in Medicine and Biology Society (EMBS), Orlando, FL, USA, 16-20 August 2016; pp. 2672-2675.

13. Khosrow-khavar, F.; Tavakolian, K.; Menon, C. Moving toward Automatic and Standalone Delineation of Seismocardiogram Signal. In Proceedings of the 2015 37th Annual International Conference of the IEEE Engineering in Medicine and Biology Society (EMBS), Milan, Italy, 25-29 August 2015; pp. 7163-7166.

14. Choudhary, T.; Bhuyan, M.K.; Sharma, L.N. A Novel Method for Aortic Valve Opening Phase Detection Using SCG Signal. IEEE Sens. J. 2020, 20, 899-908. [CrossRef]

15. Khosrow-Khavar, F.; Tavakolian, K.; Blaber, A.; Menon, C. Automatic and Robust Delineation of the Fiducial Points of the Seismocardiogram Signal for Noninvasive Estimation of Cardiac Time Intervals. IEEE Trans. Biomed. Eng. 2017, 64, 1701-1710. [CrossRef]

16. Choudhary, T.; Sharma, L.N.; Bhuyan, M.K. Automatic Detection of Aortic Valve Opening Using Seismocardiography in Healthy Individuals. IEEE J. Biomed. Health Inform. 2019, 23, 1032-1040. [CrossRef]

17. Yang, C.; Tang, S.; Tavassolian, N. Utilizing Gyroscopes towards the Automatic Annotation of Seismocardiograms. IEEE Sens. J. 2017, 17, 2129-2136. [CrossRef]

18. Wahlstrom, J.; Skog, I.; Handel, P.; Khosrow-khavar, F.; Tavakolian, K.; Stein, P.K.; Nehorai, A. A Hidden Markov Model for Seismocardiography. IEEE Trans. Biomed. Eng. 2017, 64, 2361-2372. [CrossRef] [PubMed]

19. Luu, L.; Dinh, A. Using Moving Average Method to Recognize Systole and Diastole on Seismocardiogram without ECG Signal. In Proceedings of the 2018 40th Annual International Conference of the IEEE Engineering in Medicine and Biology Society (EMBS), Honolulu, HI, USA, 17-21 July 2018; pp. 3796-3799.

20. Seppälä, E. Jatkuva-Aikainen Vitaalitoimintojen Monitorointi Pienellä Lapsilla Käyttäen Rinnalle Asetettavaa Sensoria. Master's Thesis, Univeristy of Oulu, Oulu, Finland, 16 March 2020.

21. Myllylä, T.; Elseoud, A.A.; Sorvoja, H.; Myllylä, R.A.; Harja, J.M.; Nikkinen, J.; Tervonen, O.; Kiviniemi, V. Fibre Optic Sensor for Non-Invasive Monitoring of Blood Pressure during MRI Scanning. J. Biophoton. 2011, 4, 98-107. [CrossRef] [PubMed]

22. Myllylä, T.; Zacharias, N.; Korhonen, V.; Zienkiewicz, A.; Hinrichs, H.; Kiviniemi, V.; Walter, M. Multimodal Brain Imaging with Magnetoencephalography: A Method for Measuring Blood Pressure and Cardiorespiratory Oscillations. Sci. Rep. 2017, 7, 1-9. [CrossRef]

23. Zienkiewicz, A.; Favre, M.; Ferdinando, H.; Iring, S.; Serrador, J.; Myllylä, T. Blood Pressure Wave Propagation-A Multisensor Setup for Cerebral Autoregulation Studies. Physiol. Meas. 2021, 42. [CrossRef]

24. Ngui, W.K.; Leong, M.S.; Hee, L.M.; Abdelrhman, A.M. Wavelet Analysis: Mother Wavelet Selection Methods. AMM 2013, 393, 953-958. [CrossRef]

25. Al-Qazzaz, N.; Hamid Bin Mohd Ali, S.; Ahmad, S.; Islam, M.; Escudero, J. Selection of Mother Wavelet Functions for MultiChannel EEG Signal Analysis during a Working Memory Task. Sensors 2015, 15, 29015-29035. [CrossRef]

26. Target Heart Rate Calculator. Available online: http:/ /www.stevenscreek.com/goodies/hr.shtml (accessed on 16 April 2020).

27. Lauhkonen, E.; Koponen, P.; Vuononvirta, J.; Teräsjärvi, J.; Nuolivirta, K.; Toikka, J.O.; Helminen, M.; He, Q.; Korppi, M. Gene Polymorphism of Toll-Like Receptors and Lung Function at Five to Seven Years of Age after Infant Bronchiolitis. PLoS ONE 2016, 11, e0146526. [CrossRef]

28. Wu, Z.; Luo, F.; Wu, Z.; Tao, X.; Zou, X.; Tan, J. Preoperative Exercise Facilitates Abundant Bone Marrow Collection in Patients with Type 2 Diabetes for Mononuclear Cell Therapy. Cytotherapy 2015, 17, 454-457. [CrossRef] 
29. Sporis, G.; Vucetic, V.; Jukic, I.; Omrcen, D.; Bok, D.; Custonja, Z. How Reliable Are the Equations for Predicting Maximal Heart Rate Values in Military Personnel? Mil. Med. 2011, 176, 347-351. [CrossRef]

30. Mora, N.; Cocconcelli, F.; Matrella, G.; Ciampolini, P. A Unified Methodology for Heartbeats Detection in Seismocardiogram and Ballistocardiogram Signals. Computers 2020, 9, 41. [CrossRef]

31. Furtner, M.; Staudacher, M.; Frauscher, B.; Brandauer, E.; Esnaola y Rojas, M.M.; Gschliesser, V.; Poewe, W.; Schmidauer, C.; Ritsch-Marte, M.; Högl, B. Cerebral Vasoreactivity Decreases Overnight in Severe Obstructive Sleep Apnea Syndrome: A Study of Cerebral Hemodynamics. Sleep Med. 2009, 10, 875-881. [CrossRef] [PubMed] 